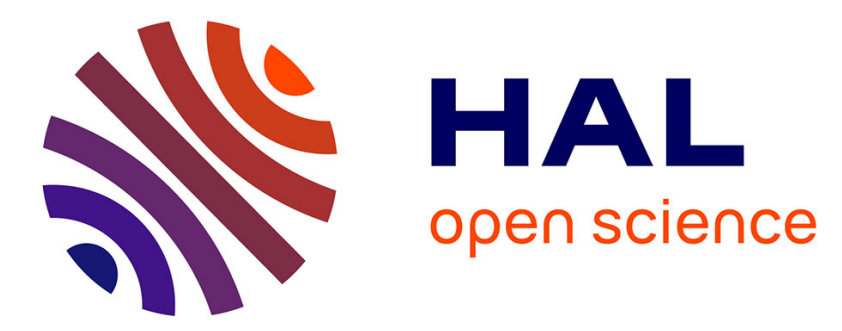

\title{
Constructing analysis-suitable parameterization of computational domain from CAD boundary by variational harmonic method
}

Gang Xu, Bernard Mourrain, Régis Duvigneau, André Galligo

\section{- To cite this version:}

Gang Xu, Bernard Mourrain, Régis Duvigneau, André Galligo. Constructing analysis-suitable parameterization of computational domain from CAD boundary by variational harmonic method. Journal of Computational Physics, 2013, 252, pp.275-289. 10.1016/j.jcp.2013.06.029 . hal-00836413

\section{HAL Id: hal-00836413 \\ https://hal.inria.fr/hal-00836413}

Submitted on 20 Jun 2013

HAL is a multi-disciplinary open access archive for the deposit and dissemination of scientific research documents, whether they are published or not. The documents may come from teaching and research institutions in France or abroad, or from public or private research centers.
L'archive ouverte pluridisciplinaire HAL, est destinée au dépôt et à la diffusion de documents scientifiques de niveau recherche, publiés ou non, émanant des établissements d'enseignement et de recherche français ou étrangers, des laboratoires publics ou privés. 


\title{
Constructing analysis-suitable parameterization of computational domain from CAD boundary by variational harmonic method
}

\author{
Gang $\mathrm{Xu}^{\mathrm{a}, \mathrm{b}, *}$, Bernard Mourrain ${ }^{\mathrm{b}}$, Régis Duvigneau ${ }^{\mathrm{c}}$, André Galligo $^{\mathrm{d}}$ \\ ${ }^{a}$ College of Computer Science, Hangzhou Dianzi University, Hangzhou 310018, P.R.China \\ ${ }^{b}$ Galaad, INRIA Sophia-Antipolis, 2004 Route des Lucioles, 06902 Cedex, France \\ ${ }^{c}$ OPALE, INRIA Sophia-Antipolis, 2004 Route des Lucioles, 06902 Cedex, France \\ ${ }^{d}$ University of Nice Sophia-Antipolis, 06108 Nice Cedex 02, France
}

\begin{abstract}
In isogeometric analysis, parameterization of computational domain has great effects as mesh generation in finite element analysis. In this paper, based on the concept of harmonic mapping from the computational domain to parametric domain, a variational harmonic approach is proposed to construct analysis-suitable parameterization of computational domain from CAD boundary for 2D and 3D isogeometric applications. Different from the previous elliptic mesh generation method in finite element analysis, the proposed method focus on isogeometric version, and converts the elliptic PDE into a nonlinear optimization problem, in which a regular term is integrated into the optimization formulation to achieve more uniform and orthogonal iso-parametric structure near convex (concave) parts of the boundary. Several examples are presented to show the efficiency of the proposed method in 2D and 3D isogeometric analysis.
\end{abstract}

Keywords: isogeometric analysis; grid generation; harmonic mapping; variational method ; analysis-suitable parameterization

\footnotetext{
${ }^{*}$ Corresponding author.

Email addresses: xugangzju@gmail.com; gxu@hdu.edu.cn (Gang Xu ), Bernard.Mourrain@inria.fr (Bernard Mourrain), Regis.Duvigneau@inria.fr (Régis Duvigneau), galligo@unice.fr (André Galligo) 


\section{Introduction}

The isogeometric analysis (IGA for short) method proposed by Hughes et al. in [19] is a new computational approach that offers the possibility of seamless integration between CAD and CAE. The method uses the same type of mathematical representation (spline representation), both for the geometry and for the physical solutions, and thus avoids this costly forth and back transformations. Moreover its reduces the number of parameters needed to describe the geometry, which is of particular interest for shape optimization.

Mesh generation, which generates a discrete grid of a computational domain from a given CAD object, is a key and the most time-consuming step in finite element analysis (FEA for short). It consumes about $80 \%$ of the overall design and analysis process [6] in aerospace, automotive and ship industry. Parametrization of computational domain in IGA, which corresponds to the mesh generation in FEA, also has some impact on analysis result and efficiency. In particular, arbitrary refinements can be performed on the computational mesh in FEA, but in IGA if we compute with tensor product B-splines, we can only perform refinement operations in each parametric direction by knot insertion or degree elevation. Hence, parameterization of computational domain is also being important for IGA. As it is pointed by Cottrell et al. [9], one of the most significant challenges towards isogeometric analysis is constructing analysis-suitable parameterizations from given CAD boundary representation.

Parameterization of a computational domain in IGA is determined by control points, knot vectors and the degrees of B-spline objects. For 2D and 3D IGA problems, the knot vectors and the degree of the computational domain are determined by the given boundary curves/surfaces. That is, given boundary curves/surfaces, the quality of parameterization of computational domain is determined by the positions of inner control points. Hence, finding a good placement of the inner control points inside the computational domain, is a key issue. As the required mesh quality in FEA $[28,29,30]$, a basic requirement of the resulting parameterization for IGA is that it doesn't have self-intersections, so that it is an injective map from the parametrization domain to the computational domain.

In the field of mesh generation in FEA, a general method is based on partial differential equations [27]. The grid points are the solution of an elliptic partial differential equation system with Dirichlet boundary conditions on all boundaries. There are several advantages in elliptic 
mesh generation. The theory of partial differential equations guarantees that the mapping between physical and transformed regions will be one-to-one. Another important property is the inherent smoothness in the solution of elliptic systems. A disadvantage of elliptic method is that there will be some non-uniform grid elements near convex (concave) parts of the boundary. Moreover, special treatment should be considered to achieve orthogonal mesh [32, 41, 40].

Motivated from the elliptic grid generation method in FEA, in this paper, we study the analysissuitable parameterization problem of computational domain in isogeometric analysis. Our main contributions are:

- A nonlinear optimization framework based on harmonic mapping theory is proposed to generate a analysis-suitable parameterization of computational domain without self-intersections for $2 \mathrm{D}$ and $3 \mathrm{D}$ isogeometric applications.

- A regular term is integrated into the optimization formulation to achieve more uniform and orthogonal iso-parametric structure near convex (concave) parts of the boundary.

- We test the parameterization results on $2 \mathrm{D}$ and $3 \mathrm{D}$ heat conduction problems to show the effectiveness of the proposed method.

The rest of the paper is organized as follows. Section 2 reviews the related work in IGA. Section 3 describes the variational harmonic method for analysis-suitable parameterization of 2D computational domain. The 3D case for volume parameterization is studied in Section 4. Some examples and comparisons based on the isogeometric heat conduction problem are presented in Section 5. Finally, we conclude this paper and outline future works in Section 6 .

\section{Related work}

In this section, we will review some related works in IGA and parameterization of computational domains.

IGA was firstly proposed by Hughes et al. [19] in 2005 to achieve the seamless integration of CAD and FEA. Since then, many researchers in the fields of computational mechanical and geometric computation were involved in this topic. The current work on isogeometric analysis can be classified into four categories: (1) application of IGA to various simulation problems [3, $5,11,18,12,26] ;(2)$ application of various modeling tools such as T-splines and PHT-splines in 
CAGD to IGA $[6,7,13,23,24]$; (3) accuracy and efficiency improvement of IGA framework by reparameterization and refinement operations $[1,4,8,10,35,36]$; (4) construction of analysissuitable computational domain from boundary information [1, 21, 22, 31, 36, 37, 38, 39].

The topic of this paper belongs to the forth field. From the viewpoint of graphics applications, volume parameterization of 3D models has been studied in $[20,34,33]$. As far as we know, there are only a few work on the parametrization of computational domains from the viewpoint of isogeometric applications. Martin et al. [21] proposed a method to fit a genus-0 triangular mesh by B-spline volume parameterization, based on discrete volumetric harmonic functions; this can be used to build computational domains for 3D IGA problems. A variational approach for constructing NURBS parameterization of swept volumes is proposed by Aigner et al [1]. Many free-form shapes in CAD systems, such as blades of turbines and propellers, are covered by this kind of volumes. Cohen et.al. [8] proposed the concept of analysis-aware modeling, in which the parameters of CAD models should be selected to facilitate isogeometric analysis. They also demonstrated the influence of parameterization of computational domains by several examples. Escobar et al. proposed a method to construct a trivariate T-spline volume of complex genus-zero solids for isogeometric application by using an adaptive tetrahedral meshing and mesh untangling technique [15]. However, the obtained solid T-splines have elements with negative Jacobians at the Gauss quadrature points. Zhang et al. proposed a robust and efficient algorithm to construct injective solid T-splines for genus-zero geometry from a boundary triangulation [38]. Based on the Morse theory, a volumetric parameterization method of mesh model with arbitrary topology is proposed in [31]. The above proposed methods demand a surface triangulation as input data. For the CAD boundary with spline representation, $r$-refinement method for generating optimal analysis-aware parameterization of computational domain is proposed based on shape optimization method $[35,36]$. However, it only works for specified analysis problems. In [37], a constraint optimization framework is proposed to obtain analysis-suitable volume parameterization of computational domain. Pettersen and Skytt proposed the spline volume faring method to obtain high-quality volume parameterization for isogeometric applications [25]. Zhang et al. studied the construction of conformal solid T-spline from boundary T-spline representation by octree structure and boundary offset [39]. In this paper, based on the harmonic mapping theory, we propose a general method to construct analysis-suitable parameterization of computational domain from given CAD boundary information. 


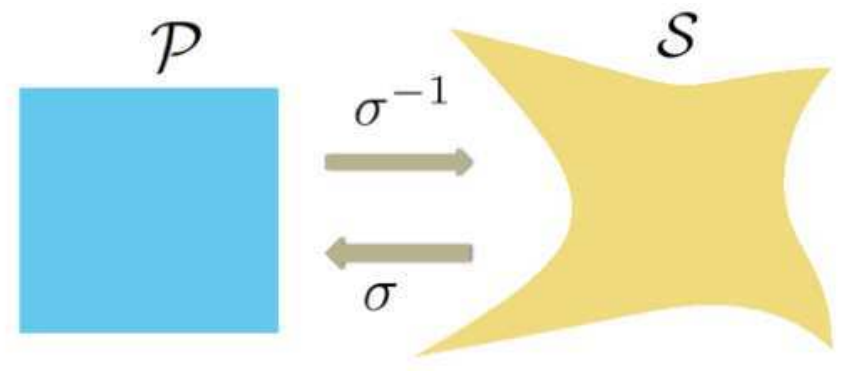

Fig. 1. The mapping $\sigma$ from physical domain $\mathcal{S}$ to parametric domain $\mathcal{P}$.

\section{Variational harmonic method for planar parameterization of $2 \mathrm{D}$ computational domain}

\subsection{Problem statement}

Consider a simply connected bounded domain $\mathcal{S}$ in two dimensional space with Cartesian coordinates $(x ; y)^{T}$. Suppose that $\mathcal{S}$ is bounded by four B-spline curves $\mathcal{S}(\xi, 0), \mathcal{S}(\xi, 1), \mathcal{S}(1, \eta)$ $\mathcal{S}(0, \eta)$. The parametric domain $\mathcal{P}$ of $\mathcal{S}$ should be a rectangular in two dimensional space with coordinates $\xi, \eta$, which is determined by the knot vector of boundary B-spline curves in $\xi$ and $\eta$ direction. The mapping from parametric space $\mathcal{P}$ to physical space $\mathcal{S}$ can be described as a B-spline surface $\mathcal{S}(\xi, \eta)=(x(\xi, \eta), y(\xi, \eta))=\sum_{i=0}^{n} \sum_{j=0}^{m} N_{i}^{p}(\xi) N_{j}^{q}(\eta) \boldsymbol{P}_{i, j}$ with given four B-spline curves as boundaries. $N_{i}^{p}(\xi)$ and $N_{j}^{q}(\eta)$ are B-spline basis functions, $\boldsymbol{P}_{i, j}$ are control points. Assume that this mapping is prescribed which maps the boundary of $\mathcal{P}$ one-to-one on the boundary of $\mathcal{S}$. The parameterization problem of computational domain can be stated as: given four boundary B-spline curves, find the placement of inner control points such that the resulting planar B-spline surface is a good computational domain for isogeometric analysis.

\subsection{Harmonic mapping and variational harmonic function}

Harmonic mapping, which is a one-to-one transformation both for 2D and 3D regions, will be used in our parameterization method. Let $\sigma: \mathcal{S} \mapsto \mathcal{P}$ be a harmonic mapping from $\mathcal{S}$ to $\mathcal{P}$. From the theory of harmonic mapping, the inverse mapping $\sigma^{-1}: \mathcal{P} \mapsto \mathcal{S}$ should be one-to-one (see Fig.1 as an example). In this section, we will convert the harmonic conditions into some constraints the inner control points of planar B-spline parameterization should satisfy. 
Suppose that $\sigma: \mathcal{S} \mapsto \mathcal{P}$ is a harmonic mapping, that is

$$
\begin{aligned}
& \Delta \xi(x, y)=\xi_{x x}+\xi_{y y}=0 \\
& \Delta \eta(x, y)=\eta_{x x}+\eta_{y y}=0
\end{aligned}
$$

By chain rules, we have

$$
\begin{aligned}
& d \xi=\xi_{x} d x+\xi_{y} d y=\xi_{x}\left(x_{\xi} d \xi+x_{\eta} d \eta\right)+\xi_{y}\left(y_{\xi} d \xi+y_{\eta} d \eta\right) \\
& d \eta=\eta_{x} d x+\eta_{y} d y=\eta_{x}\left(x_{\xi} d \xi+x_{\eta} d \eta\right)+\eta_{y}\left(y_{\xi} d \xi+y_{\eta} d \eta\right)
\end{aligned}
$$

From above two equation, we have

$$
\begin{aligned}
& \xi_{x} x_{\xi}+\xi_{y} y_{\xi}=\eta_{x} x_{\eta}+\eta_{y} y_{\eta}=1 \\
& \xi_{x} x_{\eta}+\xi_{y} y_{\eta}=\eta_{x} x_{\xi}+\eta_{y} y_{\xi}=0
\end{aligned}
$$

By solving above two linear systems, we obtain

$$
\xi_{x}=\frac{y_{\eta}}{J}, \xi_{y}=\frac{-x_{\eta}}{J}, \eta_{x}=\frac{-y_{\xi}}{J}, \eta_{y}=\frac{x_{\xi}}{J}
$$

where

$$
J=x_{\xi} y_{\eta}-x_{\eta} y_{\xi}
$$

is Jacobian of transformation from $\mathcal{P}$ to $\mathcal{S}$.

From above results and $\frac{\partial}{\partial x}=\xi_{x} \frac{\partial}{\partial \xi}, \frac{\partial}{\partial y}=\xi_{y} \frac{\partial}{\partial \xi}$, we have

$$
\begin{aligned}
& J \Delta \xi(x, y)=J \frac{\partial}{\partial x} \xi_{x}+J \frac{\partial}{\partial y} \xi_{y} \\
= & \left(J \xi_{x} \frac{\partial}{\partial \xi}+J \eta_{x} \frac{\partial}{\partial \eta}\right) \xi_{x}+\left(J \xi_{y} \frac{\partial}{\partial \xi}+J \eta_{y} \frac{\partial}{\partial \eta}\right) \xi_{y} \\
= & \left(y_{\eta} \frac{\partial}{\partial \xi}-y_{\xi} \frac{\partial}{\partial \eta}\right)\left(\frac{y_{\xi}}{J}\right)+\left(-x_{\eta} \frac{\partial}{\partial \xi}+x_{\xi} \frac{\partial}{\partial \eta}\right)\left(\frac{-x_{\eta}}{J}\right)=0
\end{aligned}
$$

After differential computation, we have

$$
-y_{\eta} L x+x_{\eta} L y=0
$$

where

$$
L=\left(x_{\eta}^{2}+y_{\eta}^{2}\right) \frac{\partial^{2}}{\partial \xi^{2}}-2\left(x_{\xi} x_{\eta}+y_{\xi} y_{\eta}\right) \frac{\partial^{2}}{\partial \xi \eta}+\left(x_{\xi}^{2}+y_{\xi}^{2}\right) \frac{\partial^{2}}{\partial \eta^{2}}
$$


Similarly, from $J \Delta \eta(x, y)=0$, we have

$$
y_{\xi} L x-x_{\xi} L y=0
$$

Then we obtain

$$
L x(\xi, \eta)=L y(\xi, \eta)=0
$$

that is,

$$
\|L \mathcal{S}(\xi, \eta)\|^{2}=(L x)^{2}+(L y)^{2}=0
$$

This is a nonlinear system in terms of inner control points of the planar B-spline parameterization. The final grid obtained by this method usually has non-uniform elements near convex (concave) boundary region. In order to solve this problem, we minimize the following energy function, which is related to the uniformity of the iso-parametric net on the B-spline surface,

$$
\iint\left\|\mathcal{S}_{\xi \xi}\right\|^{2}+\left\|\mathcal{S}_{\eta \eta}\right\|^{2}+2\left\|\mathcal{S}_{\xi \eta}\right\|^{2} d \xi d \eta
$$

Moreover, orthogonality is also an important property for analysis-suitable parameterization in numerical simulation $[40,32]$. In order to achieve an iso-parametric structure with good orthogonality, we minimize the following energy function as in [36]

$$
\iint\left\|\mathcal{S}_{\xi}\right\|^{2}+\left\|\mathcal{S}_{\eta}\right\|^{2} d \xi d \eta
$$

Then by adding the harmonic mapping term into above two energy functions, we obtain a new energy function for parameterization of computational domain

$$
\begin{aligned}
& \iint\|L \mathcal{S}(\xi, \eta)\|^{2}+\lambda_{1}\left(\left\|\mathcal{S}_{\xi \xi}\right\|^{2}+\left\|\mathcal{S}_{\eta \eta}\right\|^{2}\right. \\
& \left.+2\left\|\mathcal{S}_{\xi \eta}\right\|^{2}\right)+\lambda_{2}\left(\left\|\mathcal{S}_{\xi}\right\|^{2}+\left\|\mathcal{S}_{\eta}\right\|^{2}\right) d \xi d \eta
\end{aligned}
$$

where $\lambda_{1}$ and $\lambda_{2}$ are positive weights to control the final parameterization results.

Remark 3.1. For different choice of $\lambda_{1}$ and $\lambda_{2}$ in the objective function (3), different parameterization results will be achieved. If $\lambda_{1}>\lambda_{2}$, then the resulting iso-parametric structure has better uniformity. If $\lambda_{2}>\lambda_{1}$, then we can obtain an iso-parametric grid with better orthogonality. See Fig. 3 for an example to show the effects of the positive weights $\lambda_{1}$ and $\lambda_{2}$.

The Discrete Coons method is used to construct initial inner control points, and the Steepest Descent method is employed to minimize the energy function. The details will be described in the following two subsections. 


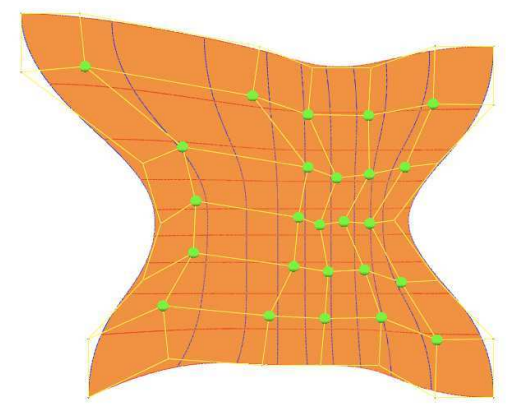

(a)

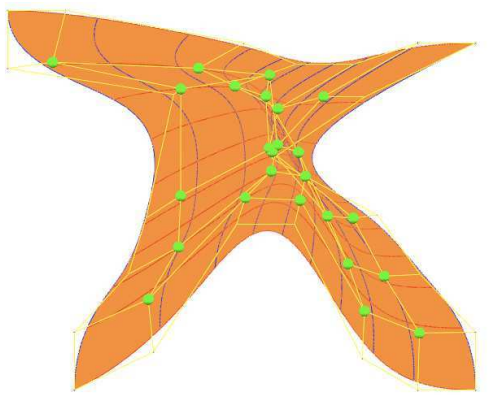

(b)

Fig. 2. Examples of B-spline surface constructed by discrete Coons method: (a) example without self-intersection ; (b) example with self-intersections. Green control points are inner control points to be constructed.

\subsection{Initial construction of inner control points}

In order to solve this constraint optimization problem, an initial construction of inner control points is required. We rely on the discrete Coons method presented in [16] to generate inner control points as initial value from boundary control points. See Fig. 2 (a) for an example.

Given the boundary control points $\boldsymbol{P}_{0, j}, \boldsymbol{P}_{n, j}, \boldsymbol{P}_{i, 0}, \boldsymbol{P}_{i, m}, i=0, \ldots, n, j=0, \ldots, m$, the inner control points $\boldsymbol{P}_{i, j}(i=1, \ldots, n-1, j=1, \ldots, m-1)$ can be constructed by the discrete Coons method as follows:

$$
\begin{aligned}
\boldsymbol{P}_{i, j}= & \left(1-\frac{i}{n}\right) \boldsymbol{P}_{0, j}+\frac{i}{n} \boldsymbol{P}_{n, j}+\left(1-\frac{j}{m}\right) \boldsymbol{P}_{i, 0}+\frac{j}{m} \boldsymbol{P}_{i, m} \\
& -\left[\begin{array}{ll}
1-\frac{i}{n} & \frac{i}{n}
\end{array}\right]\left[\begin{array}{ll}
\boldsymbol{P}_{0,0} & \boldsymbol{P}_{0, m} \\
\boldsymbol{P}_{n, 0} & \boldsymbol{P}_{n, m}
\end{array}\right]\left[\begin{array}{c}
1-\frac{j}{m} \\
\frac{j}{m}
\end{array}\right]
\end{aligned}
$$

Since the sum of the coefficients equals 1 , the resulting inner control points lie in the convex hull of the boundary control points. For some given boundary curves, this construction may cause some self-intersections, and lead to an improper parameterization for IGA. See Fig. 2 (b) for such an example.

\subsection{Optimization method}

In the proposed approach, we minimize the objective function (3), by moving inner control points of the computational domain. Therefore, we consider as optimization variables the 
coordinates of the inner control points and as cost function the error of the IGA solution. The optimization algorithm used for this study is a classical steepest-descent method in conjunction with a back-tracking line-search. For this exercise, the gradient of the cost function is approximated using a centered finite-differencing scheme.

Each iteration $k$ of the optimization algorithm can be summarized as follows, starting from a point $x_{k}$ in the variable space:

1. Evaluation of perturbed points $x_{k}+\epsilon e_{k}$

2. Estimation of the gradient $\nabla f\left(x_{k}\right)$ by finite-difference

3. Define search direction $d_{k}=-\nabla f\left(x_{k}\right)$

4. Line search : find $\rho$ such as $f\left(x_{k}+\rho d_{k}\right)<f\left(x_{k}\right)$

These steps are carried out until a stopping criterion is satisfied.

\subsection{Overview of variational harmonic method}

In general, the proposed variational harmonic method can be summarized as follows:

Input: four coplanar boundary B-spline curves

Output: inner control points and the corresponding planar B-spline surfaces

- Construct the initial inner control points by discrete Coons method as in subsection 3.3;

- Solve the following optimization problem by using steepest-descent method

$$
\begin{aligned}
& \operatorname{Min} \iint\|L \mathcal{S}(\xi, \eta)\|^{2}+\lambda_{1}\left(\left\|\mathcal{S}_{\xi \xi}\right\|^{2}+\left\|\mathcal{S}_{\eta \eta}\right\|^{2}\right. \\
& \left.+2\left\|\mathcal{S}_{\xi \eta}\right\|^{2}\right)+\lambda_{2}\left(\left\|\mathcal{S}_{\xi}\right\|^{2}+\left\|\mathcal{S}_{\eta}\right\|^{2}\right) d \xi d \eta
\end{aligned}
$$

- Generate the corresponding planar B-spline surface $\mathcal{S}(\xi, \eta)$ as computational domain.

\section{Variational harmonic method for volume parameterization of $3 \mathrm{D}$ computational domain}

In this section, we will study the volume parameterization problem by variational harmonic method. Suppose that $\mathcal{S}$ is a simply connected bounded domain in three dimensional space with Cartesian coordinates $(x ; y ; z)^{T}$, and is bounded by six B-spline surfaces $\mathcal{S}(\xi, 0, \zeta), \mathcal{S}(\xi, 1, \zeta)$, 
$\mathcal{S}(1, \eta, \zeta), \mathcal{S}(0, \eta, \zeta), \mathcal{S}(\xi, \eta, 0), \mathcal{S}(\xi, \eta, 1)$. The parametric domain $\mathcal{P}$ of $\mathcal{S}$ should be a cube in three dimensional space with coordinates $\xi, \eta, \zeta$, which is determined by the knot vector of boundary B-spline surfaces in $\xi, \eta$ and $\zeta$ direction. The mapping from parametric space $\mathcal{P}$ to physical space $\mathcal{S}$ can be described as a B-spline volume $\mathcal{S}(\xi, \eta, \zeta)=(x(\xi, \eta, \zeta), y(\xi, \eta, \zeta), z(\xi, \eta, \zeta))=$ $\sum_{i=0}^{n} \sum_{j=0}^{m} \sum_{k=0}^{l} N_{i}^{p}(\xi) N_{j}^{q}(\eta) N_{k}^{r}(\zeta) \boldsymbol{P}_{i, j, k}$ with given six B-spline surfaces as boundaries. $N_{i}^{p}(\xi)$, $N_{j}^{q}(\eta)$ and $N_{k}^{r}(\zeta)$ are B-spline basis functions, $\boldsymbol{P}_{i, j, k}$ are control points. Assume that this mapping is prescribed which maps the boundary of $\mathcal{P}$ one-to-one on the boundary of $\mathcal{S}$. The volume parameterization problem of 3D computational domain can be stated as: given six boundary Bspline surfaces, find the placement of inner control points such that the resulting trivariate B-spline parametric volume is a good computational domain for 3D isogeometric analysis.

\subsection{Harmonic mapping and variational harmonic function}

Similar with the 2D case, the harmonic conditions in 3D case will be converted into some constraints the inner control points of trivariate B-spline volume parameterization should satisfy.

If the mapping $\sigma: \mathcal{S} \mapsto \mathcal{P}$ is a harmonic mapping, we have

$$
\begin{aligned}
\Delta \xi(x, y, z) & =\xi_{x x}+\xi_{y y}+\xi_{z z}=0 \\
\Delta \eta(x, y, z) & =\eta_{x x}+\eta_{y y}+\eta_{z z}=0 \\
\Delta \zeta(x, y, z) & =\zeta_{x x}+\zeta_{y y}+\zeta_{z z}=0
\end{aligned}
$$

By chain rules, we have

$$
\begin{aligned}
& d \xi=\xi_{x} d x+\xi_{y} d y+\xi_{z} d z=\xi_{x}\left(x_{\xi} d \xi+x_{\eta} d \eta+x_{\zeta} d \zeta\right) \\
& +\xi_{y}\left(y_{\xi} d \xi+y_{\eta} d \eta+y_{\zeta} d \zeta\right)+\xi_{z}\left(z_{\xi} d \xi+z_{\eta} d \eta+z_{\zeta} d \zeta\right) \\
& d \eta=\eta_{x} d x+\eta_{y} d y+\eta_{z} d z=\eta_{x}\left(x_{\xi} d \xi+x_{\eta} d \eta+x_{\zeta} d \zeta\right) \\
& +\eta_{y}\left(y_{\xi} d \xi+y_{\eta} d \eta+y_{\zeta} d \zeta\right)+\eta_{z}\left(z_{\xi} d \xi+z_{\eta} d \eta+z_{\zeta} d \zeta\right) \\
& d \zeta=\zeta_{x} d x+\zeta_{y} d y+\zeta_{z} d z=\zeta_{x}\left(x_{\xi} d \xi+x_{\eta} d \eta+x_{\zeta} d \zeta\right) \\
& +\zeta_{y}\left(y_{\xi} d \xi+y_{\eta} d \eta+y_{\zeta} d \zeta\right)+\zeta_{z}\left(z_{\xi} d \xi+z_{\eta} d \eta+z_{\zeta} d \zeta\right)
\end{aligned}
$$


From above three equation, we have

$$
\begin{aligned}
\xi_{x} x_{\xi}+\xi_{y} y_{\xi}+\xi_{z} z_{\xi} & =1 \\
\eta_{x} x_{\eta}+\eta_{y} y_{\eta}+\eta_{z} z_{\eta}=\zeta_{x} x_{\zeta}+\zeta_{y} y_{\zeta}+\zeta_{z} z_{\zeta} & =1 \\
\xi_{x} x_{\eta}+\xi_{y} y_{\eta}+\xi_{z} z_{\eta}=\eta_{x} x_{\xi}+\eta_{y} y_{\xi}+\eta_{z} z_{\xi} & =0 \\
\xi_{x} x_{\zeta}+\xi_{y} y_{\zeta}+\xi_{z} z_{\zeta}=\zeta_{x} x_{\xi}+\zeta_{y} y_{\xi}+\zeta_{z} z_{\xi} & =0 \\
\eta_{x} x_{\zeta}+\eta_{y} y_{\zeta}+\eta_{z} z_{\zeta}=\zeta_{x} x_{\eta}+\zeta_{y} y_{\eta}+\zeta_{z} z_{\eta} & =0
\end{aligned}
$$

By solving above three linear systems, we obtain

$$
\begin{gathered}
\xi_{x}=\frac{y_{\eta} z_{\zeta}-y_{\zeta} z_{\eta}}{J}, \xi_{y}=-\frac{x_{\eta} z_{\zeta}-x_{\zeta} z_{\eta}}{J}, \xi_{z}=\frac{y_{\eta} z_{\zeta}-y_{\zeta} z_{\eta}}{J}, \\
\eta_{x}=\frac{y_{\xi} z_{\zeta}-y_{\zeta} z_{\xi}}{J}, \eta_{y}=-\frac{x_{\xi} z_{\zeta}-x_{\zeta} z_{\xi}}{J}, \eta_{z}=\frac{y_{\xi} z_{\zeta}-y_{\zeta} z_{\xi}}{J}, \\
\zeta_{x}=\frac{y_{\eta} z_{\xi}-y_{\xi} z_{\eta}}{J}, \zeta_{y}=-\frac{x_{\eta} z_{\xi}-x_{\xi} z_{\eta}}{J}, \zeta_{z}=\frac{y_{\eta} z_{\xi}-y_{\xi} z_{\eta}}{J},
\end{gathered}
$$

where

$$
J=\left|\begin{array}{ccc}
x_{\xi} & y_{\xi} & z_{\xi} \\
x_{\eta} & y_{\eta} & z_{\eta} \\
x_{\zeta} & y_{\zeta} & z_{\zeta}
\end{array}\right|
$$

is Jacobian of transformation from $\mathcal{P}$ to $\mathcal{S}$.

From above results and $J \Delta \xi(x, y, z)=0, J \Delta \eta(x, y, z)=0, J \Delta \zeta(x, y, z)=0$, the following condition can be derived,

$$
L x(\xi, \eta, \zeta)=L y(\xi, \eta, \zeta)=L z(\xi, \eta, \zeta)=0
$$

where

$$
\begin{gathered}
L=a^{11} \frac{\partial^{2}}{\partial \xi^{2}}+2 a^{12} \frac{\partial^{2}}{\partial \xi \eta}+2 a^{13} \frac{\partial^{2}}{\partial \xi \zeta}+a^{22} \frac{\partial^{2}}{\partial \eta^{2}}+2 a^{23} \frac{\partial^{2}}{\partial \eta \zeta}+a^{33} \frac{\partial^{2}}{\partial \zeta^{2}}, \\
a^{11}=a_{22} a_{33}-a_{23}^{2}, a^{12}=a_{13} a_{23}-a_{12} a_{33}, a^{13}=a_{12} a_{23}-a_{13} a_{22}, \\
a^{22}=a_{11} a_{33}-a_{13}^{2}, a^{23}=a_{13} a_{12}-a_{11} a_{23}, a^{33}=a_{11} a_{22}-a_{12}^{2},
\end{gathered}
$$

and

$$
\begin{aligned}
& a_{11}=\left(\mathcal{S}_{\xi}, \mathcal{S}_{\xi}\right), a_{12}=\left(\mathcal{S}_{\xi}, \mathcal{S}_{\eta}\right), a_{13}=\left(\mathcal{S}_{\xi}, \mathcal{S}_{\zeta}\right) \\
& a_{22}=\left(\mathcal{S}_{\eta}, \mathcal{S}_{\eta}\right), a_{23}=\left(\mathcal{S}_{\eta}, \mathcal{S}_{\zeta}\right), a_{33}=\left(\mathcal{S}_{\zeta}, \mathcal{S}_{\zeta}\right) .
\end{aligned}
$$


Eq. (4) can be rewritten as

$$
\|L \mathcal{S}(\xi, \eta, \zeta)\|^{2}=(L x)^{2}+(L y)^{2}+(L z)^{2}=0
$$

Similarly with the $2 \mathrm{D}$ problem, it is also a nonlinear system in terms of inner control points of the B-spline volume. In order to achieve uniform and orthogonal iso-parametric structure near convex(concave) boundary region, we minimize the following energy function as in [37]

$$
\begin{aligned}
& \iint \lambda_{1}\left(\left\|\mathcal{S}_{\xi \xi}\right\|^{2}+\left\|\mathcal{S}_{\eta \eta}\right\|^{2}+\left\|\mathcal{S}_{\zeta \zeta}\right\|^{2}+2\left\|\mathcal{S}_{\xi \eta}\right\|^{2}+2\left\|\mathcal{S}_{\eta \zeta}\right\|^{2}+2\left\|\mathcal{S}_{\xi \zeta}\right\|^{2}\right) \\
& +\lambda_{2}\left(\left\|\mathcal{S}_{\xi}\right\|^{2}+\left\|\mathcal{S}_{\eta}\right\|^{2}+\left\|\mathcal{S}_{\zeta}\right\|^{2}\right) d \xi d \eta d \zeta
\end{aligned}
$$

in which the first part with coefficient $\lambda_{1}$ is related to the uniformity of the iso-parametric structure, and the second part with coefficient $\lambda_{2}$ is related to the orthogonality of the iso-parametric structure.

A new energy function for volume parameterization of computational domain can be obtained by combining the harmonic mapping term and the above energy function as follows,

$$
\begin{aligned}
& \iint\|L \mathcal{S}(\xi, \eta, \zeta)\|^{2}+\lambda_{1}\left(\left\|\mathcal{S}_{\xi \xi}\right\|^{2}+\left\|\mathcal{S}_{\eta \eta}\right\|^{2}+\left\|\mathcal{S}_{\zeta \zeta}\right\|^{2}\right. \\
& \left.+2\left\|\mathcal{S}_{\xi \eta}\right\|^{2}+2\left\|\mathcal{S}_{\eta \zeta}\right\|^{2}+2\left\|\mathcal{S}_{\xi \zeta}\right\|^{2}\right) \\
& +\lambda_{2}\left(\left\|\mathcal{S}_{\xi}\right\|^{2}+\left\|\mathcal{S}_{\eta}\right\|^{2}+\left\|\mathcal{S}_{\zeta}\right\|^{2}\right) d \xi d \eta d \zeta
\end{aligned}
$$

where $\lambda_{1}$ and $\lambda_{2}$ are positive weights to control the final parameterization results.

Remark 4.1. The weights $\lambda_{1}$ and $\lambda_{2}$ have similar effects on the volume parameterization results as stated in Remark 3.1.

\subsection{Initial construction of inner control points}

The initial construction of inner control points for volume parameterization is based on the discrete Coons volume generation method [35], which can be considered as the trivariate generalization of the discrete Conns patches presented in [16].

Suppose that the given boundary surfaces are B-spline surfaces and that the opposite boundary B-spline surfaces have the same degree, number of control points and knot vectors. The interior control points $\boldsymbol{P}_{i, j, k}$ can be constructed as linear combination of boundary control points according to the following formulas: 


$$
\begin{aligned}
& \boldsymbol{P}_{i, j, k}=(1-i / l) \boldsymbol{P}_{0, j, k}+i / l \boldsymbol{P}_{l, j, k}+(1-j / m) \boldsymbol{P}_{i, 0, k} \\
& +j / m \boldsymbol{P}_{i, m, k}+(1-k / n) \boldsymbol{P}_{i, j, 0}+k / n \boldsymbol{P}_{i, j, n} \\
& -[1-i / l, i / l]\left[\begin{array}{rr}
\boldsymbol{P}_{0,0, k} & \boldsymbol{P}_{0, m, k} \\
\boldsymbol{P}_{l, 0, k} & \boldsymbol{P}_{l, m, k}
\end{array}\right]\left[\begin{array}{c}
1-j / m \\
j / m
\end{array}\right] \\
& -[1-j / m, j / m]\left[\begin{array}{ll}
\boldsymbol{P}_{i, 0,0} & \boldsymbol{P}_{i, 0, n} \\
\boldsymbol{P}_{i, m, 0} & \boldsymbol{P}_{i, m, n}
\end{array}\right]\left[\begin{array}{c}
1-k / n \\
k / n
\end{array}\right] \\
& -[1-k / n, k / n]\left[\begin{array}{ll}
\boldsymbol{P}_{0, j, 0} & \boldsymbol{P}_{l, j, 0} \\
\boldsymbol{P}_{0, j, n} & \boldsymbol{P}_{l, j, n}
\end{array}\right]\left[\begin{array}{c}
1-i / l \\
i / l
\end{array}\right] \\
& \left.+(1-k / n)[1-i / l, i / l]\left[\begin{array}{cc}
\boldsymbol{P}_{0,0,0} & \boldsymbol{P}_{0, m, 0} \\
\boldsymbol{P}_{l, 0,0} & \boldsymbol{P}_{l, m, 0}
\end{array}\right]\left[\begin{array}{c}
1-j / m \\
j / m
\end{array}\right]\right] \\
& \left.+k / n[1-i / l, i / l]\left[\begin{array}{cc}
\boldsymbol{P}_{0,0, n} & \boldsymbol{P}_{0, m, n} \\
\boldsymbol{P}_{l, 0, n} & \boldsymbol{P}_{l, m, n}
\end{array}\right]\left[\begin{array}{c}
1-j / m \\
j / m
\end{array}\right]\right]
\end{aligned}
$$

Then the corresponding B-spline volume has the following form

$$
\mathcal{S}(\xi, \eta, \zeta)=\sum_{i=0}^{l} \sum_{j=0}^{m} \sum_{k=0}^{n} N_{i}^{p}(\xi) N_{j}^{q}(\eta) N_{k}^{r}(\zeta) \boldsymbol{P}_{i, j, k} .
$$

where $N_{i}^{p}(\xi), N_{j}^{q}(\eta)$ and $N_{k}^{r}(\zeta)$ are B-spline basis functions given by the boundary surfaces.

\subsection{Overview of volume parameterization method}

The proposed variational harmonic method for volume parameterization can be summarized as follows:

Input: six boundary B-spline surfaces

Output: inner control points and the corresponding B-spline volumes

- Construct the initial inner control points by discrete Coons method as in subsection 4.2;

- Solve the following optimization problem by using steepest-descent method presented in ;

$$
\begin{aligned}
& \operatorname{Min} \iint\|L \mathcal{S}(\xi, \eta, \zeta)\|^{2}+\lambda_{1}\left(\left\|\mathcal{S}_{\xi \xi}\right\|^{2}+\left\|\mathcal{S}_{\eta \eta}\right\|^{2}+\left\|\mathcal{S}_{\zeta \zeta}\right\|^{2}\right. \\
& \left.+2\left\|\mathcal{S}_{\xi \eta}\right\|^{2}+2\left\|\mathcal{S}_{\eta \zeta}\right\|^{2}+2\left\|\mathcal{S}_{\xi \zeta}\right\|^{2}\right) \\
& +\lambda_{2}\left(\left\|\mathcal{S}_{\xi}\right\|^{2}+\left\|\mathcal{S}_{\eta}\right\|^{2}+\left\|\mathcal{S}_{\zeta}\right\|^{2}\right) d \xi d \eta d \zeta
\end{aligned}
$$

- Generate the corresponding B-spline volume $\mathcal{S}(\xi, \eta, \zeta)$ as computational domain. 


\section{Examples and comparison}

In this section, we aim at presenting several examples to show the efficiency of the proposed method. We will also give a comparison study between the original parameterization and the final parameterization constructed by the proposed method based on the $2 \mathrm{D}$ and $3 \mathrm{D}$ heat conduction problem.

\subsection{Test model - heat conduction problem}

For ease of presentation, we consider the second order elliptic PDE with homogeneous Dirichlet boundary condition as an illustrative model problem :

$$
\begin{array}{rlrl}
-\Delta U(\mathbf{x}) & =f(\mathbf{x}) & & \text { in } \Omega \\
U(\mathbf{x}) & =0 & \text { on } \partial \Omega
\end{array}
$$

where $\mathbf{x}$ are the Cartesian coordinates, $\Omega$ is a Lipschitz domain with boundary $\partial \Omega, f(\mathbf{x}) \in L^{2}(\Omega)$ : $\Omega \mapsto \mathbb{R}$ is a given source term, and $U(\mathbf{x}): \Omega \mapsto \mathbb{R}$ is the unknown solution.

Starting from a planar B-spline surface (or trivariate B-spline parametric volume) as computational domain, a general framework of an isogeometric solver for 2D and 3D heat conduction problem (6) has been implemented as a plugin in the AXEL ${ }^{1}$ platform, yielding a B-spline surface (or B-spline volume) as solution field. Additional details concerning the isogeometric solver of problem (6) can be found in [14]. The proposed variational harmonic method is implemented as a part of the isogeometric toolbox of the project EXCITING ${ }^{2}$.

In this paper, we test the different parameterizations of computational domains for $2 \mathrm{D}$ heat conduction problem (6) with source term

$$
\boldsymbol{f}(x, y)=\frac{4 \pi^{2}}{9} \sin \left(\frac{\pi x}{3}\right) \sin \left(\frac{\pi y}{3}\right) .
$$

and $3 \mathrm{D}$ problem with source term

$$
\boldsymbol{f}(x, y, z)=\frac{\pi^{2}}{3} \sin \left(\frac{\pi x}{3}\right) \sin \left(\frac{\pi y}{3}\right) \sin \left(\frac{\pi z}{3}\right) .
$$

For problems with unknown exact solution $U$, suppose that $U_{h}$ is the approximation solution obtained by isogeometric method, then the discrete error $e=U-U_{h}$. We employ a posteriori error

\footnotetext{
${ }^{1}$ http://axel.inria.fr/

${ }^{2}$ http://exciting-project.eu/
} 
assessment proposed in [36] to compare the initial and final parameterization of the computational domain. It can be obtained by resolving the following problem,

$$
\begin{aligned}
& \Delta e=-f+\Delta U_{h} \quad \text { in } \Omega \\
& e=0 \quad \text { on } \partial \Omega_{D}
\end{aligned}
$$

The approximation error $e$ from (7) also has a B-spline form. Some $h$-refinement operation should be performed to achieve more accurate results for above problem. Though it is much more expensive, it can be used as an error assessment method to show the effectiveness of the proposed construction method of computational domain.

The first 2D example is shown in Fig. 3. Fig. 3 (a) presents the given boundary B-spline curves. Fig. 3(b) presents the initial parametrization of computational domain constructed by discrete Coons method. Fig. 3(c) shows the iso-parametric curves of the initial B-spline parameterization. Fig. 3(d), Fig. 3 (e) and Fig. 3(f) show the optimized parametrization of computational domain constructed by the proposed variational harmonic method with different choice of $\lambda_{1}$ and $\lambda_{2}$. The corresponding iso-parametric structure of the resulting planar B-spline parameterization are shown in Fig. 3(g), Fig. 3 (h) and Fig. 3(i) respectively. As described in Remark 3.1, the iso-parametric structure with $\lambda_{1}=0$ and $\lambda_{2}=1$ in Fig. $3(\mathrm{~g})$ has the best orthogonality, the iso-parametric net with $\lambda_{1}=1$ and $\lambda_{2}=0$ in Fig. 3(h) has the best uniformity, and a good balance between uniformity and orthogonality can be achieved in Fig. 3(i) with $\lambda_{1}=0.5$ and $\lambda_{2}=0.5$. The corresponding simulation errors of 2D heat conduction problem (7) for different parameterization shown in Fig. 3(b) and Fig. 3(f) are illustrated in Fig. 4 (a) and Fig. 4 (b) with the same scale.

Another 2D example is illustrated in Fig. 5. The given boundary B-spline curves is shown in Fig. 5 (a). Fig. 5(b) presents the initial parametrization of computational domain constructed by discrete Coons method. There are some self-intersections on the initial parameterization as shown by the iso-parametric curves in Fig. 5(d). Fig. 5(c) shows the optimized parametrization of computational domain constructed by variational harmonic method. The optimized parameterization has no self-intersection as illustrated in Fig. 5(e).

Fig. 6 shows a 3D example, which is drawn partly to illustrate the interior information of the volume. The given boundary B-spline surfaces and curves are shown in Fig. 6 (a) and Fig.6 (b). Fig. 6 (c) presents the initial volume parametrization of computational domain constructed by discrete Coons method. There are some self-intersections on the initial parameterization. Fig. 


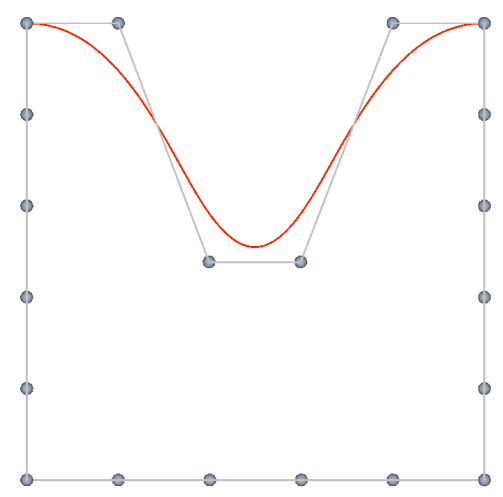

(a) boundary curves;

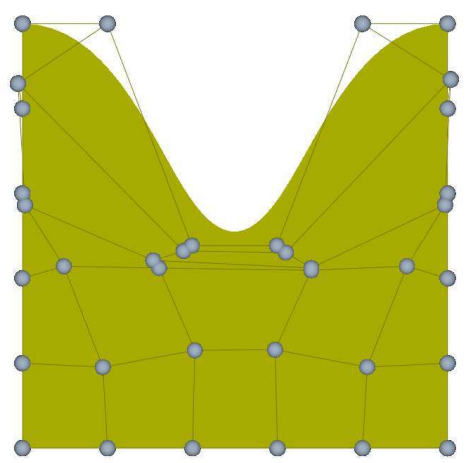

(d) optimized

parameterization with

$\lambda_{1}=0$ and $\lambda_{2}=1$;

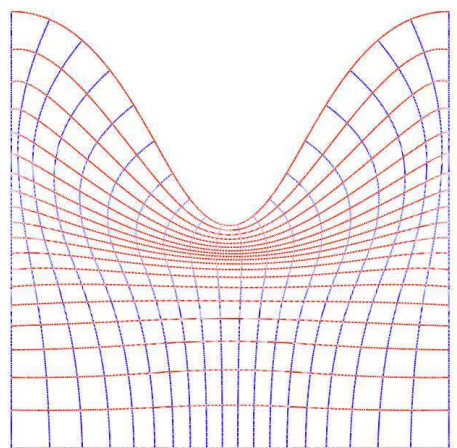

(g) optimized

parameterization with

$$
\lambda_{1}=0 \text { and } \lambda_{2}=1
$$
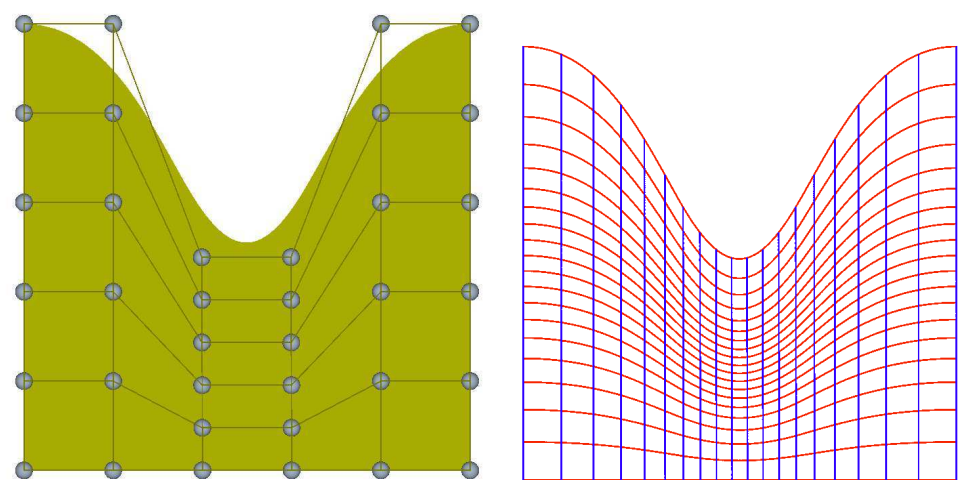

(b) initial parameterization

(c) initial iso-parametric net

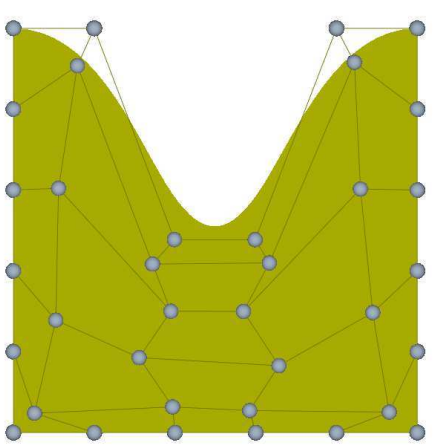

(e) optimized

parameterization with

$$
\lambda_{1}=1 \text { and } \lambda_{2}=0
$$

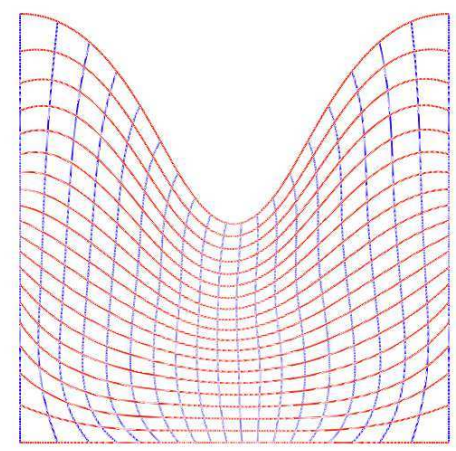

(h) optimized

parameterization with

$$
\lambda_{1}=1 \text { and } \lambda_{2}=0
$$

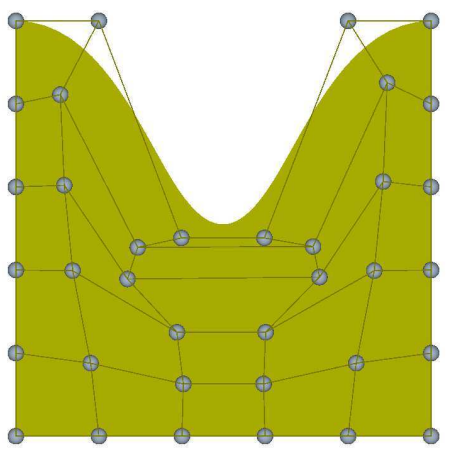

(f) optimized

parameterization with

$\lambda_{1}=0.5$ and $\lambda_{2}=0.5$

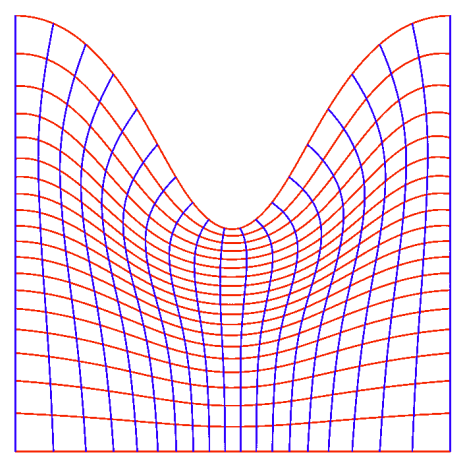

(i) optimized

parameterization with

$\lambda_{1}=0.5$ and $\lambda_{2}=0.5$

Fig. 3. 2D example I. 


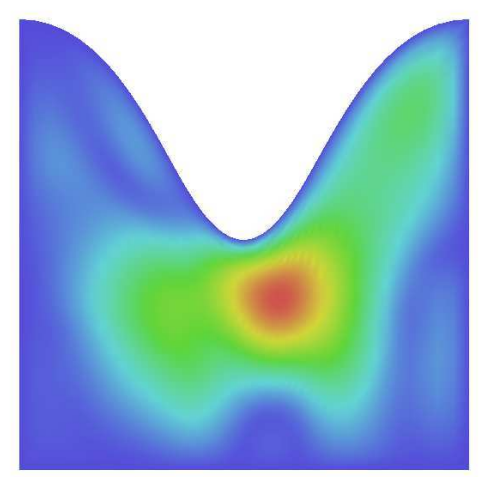

(a)

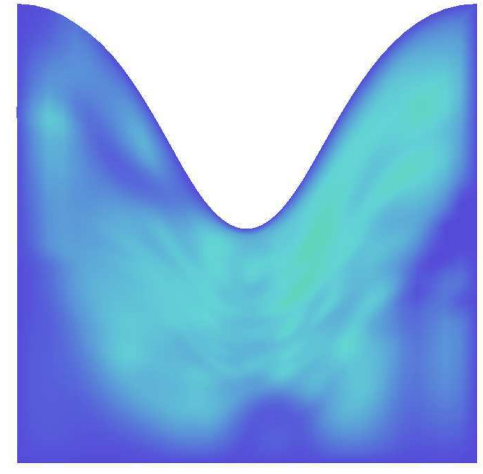

(b)

Fig. 4. Simulation comparison for $2 \mathrm{D}$ example in Fig.3. (a) simulation error w.r.t Coons parameterization in Fig.3(b); (b) simulation error w.r.t optimized parameterization in Fig.3(f) with same scale.

6 (d) shows the final volume parameterization of computational domain without self-intersections constructed by variational harmonic method. To illustrate the quality of the parameterization, the iso-parametric surfaces of the resulting B-spline volume are presented in Fig. 6 (e) and Fig.6 (f). Fig. 6 (g) and Fig.6 (h) presents the corresponding solution field obtained from the volume parameterization in Fig. 6 (c) and Fig. 6 (d) . Obviously, the solution field in Fig. 6 (h) is more smooth than the solution field in Fig. 6 (g), which indicates that the final volume parameterization is better than the initial one. Another 3D example with complex geometry is shown in Fig.7.

Quantitative data of the examples presented in Fig. 3, Fig.5, Fig. 6 and Fig. 7 are summarized in Table.1. Overall, the optimized parameterization obtained by our method has better quality, and can achieve better simulation results than the initial Coons parameterization.

Remark 5.1. The optimization problem in this paper is multi-criterion and solved by aggregating the criteria multiplied by weights $\lambda_{1}$ and $\lambda_{2}$. This straightforward approach has a well-known drawback: the criteria may have different orders of magnitude and a naive choice of the weights may yield a stronger decrease of a criterion, while others are not modified. To overcome this difficulty, one may use adaptive weights: starting from equal weights, one adapts progressively their values after some iterations, to achieve the same decrease for all criteria. 


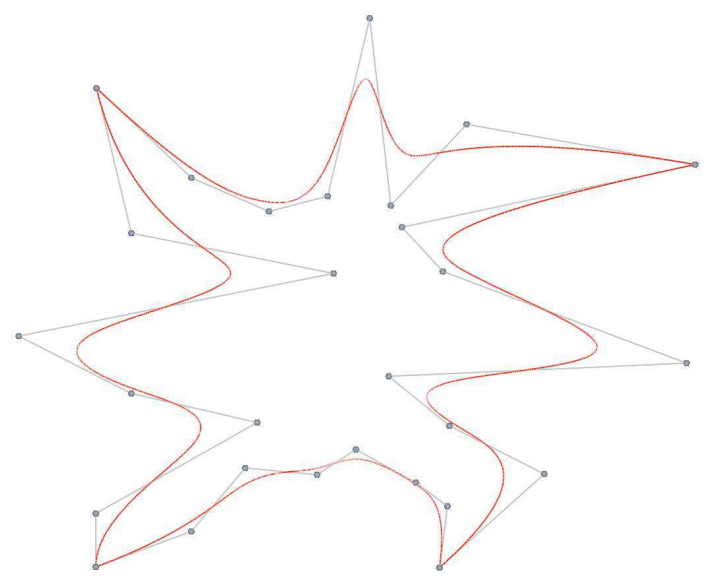

(a) boundary curves

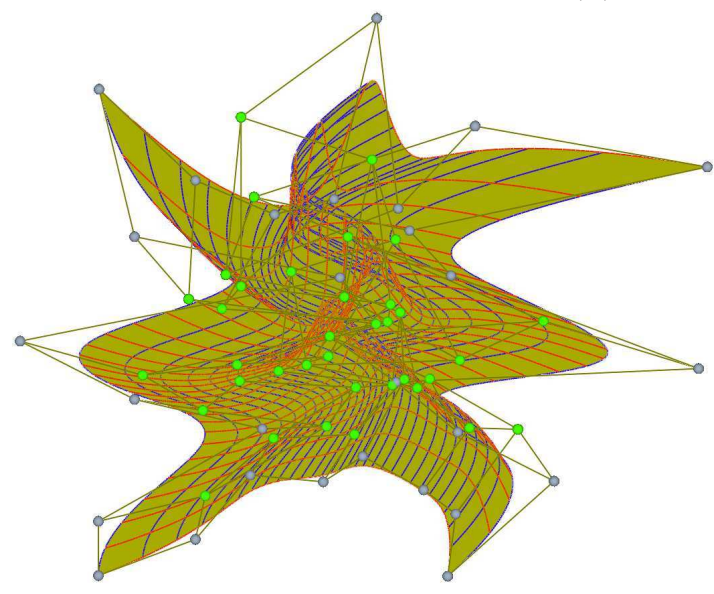

(b) initial Coons parameterization

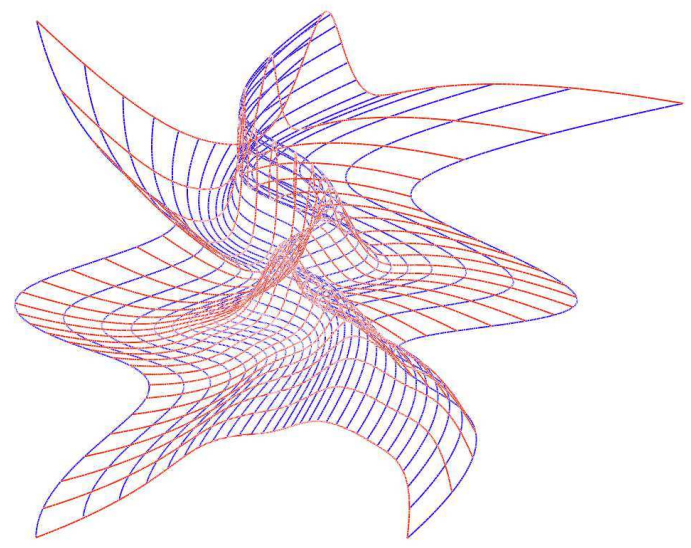

(d) initial iso-parametric net

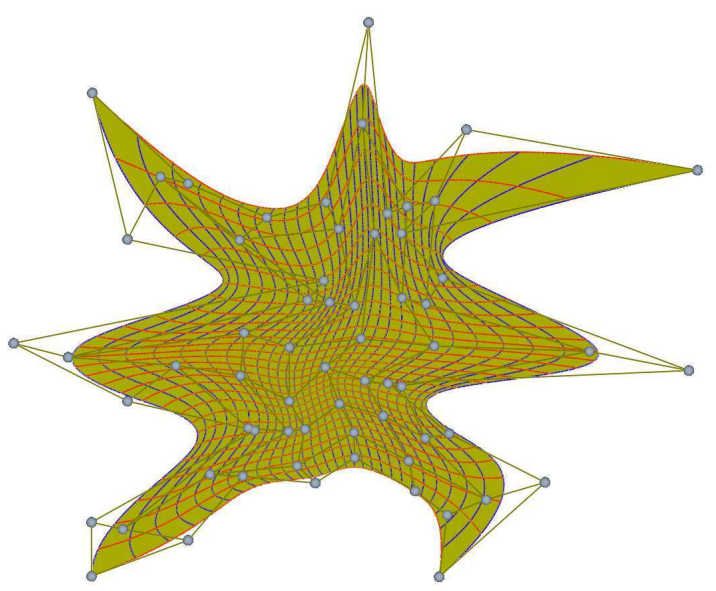

(c) optimized parameterization

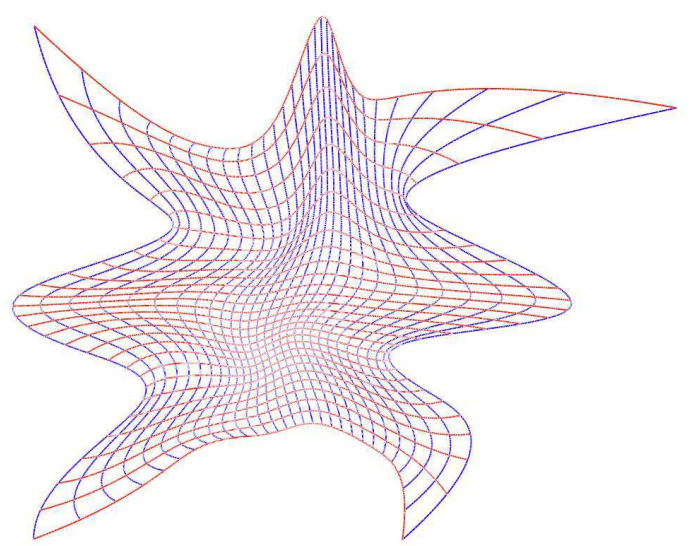

(e) optimized iso-parametric net

Fig. 5. 2D example II. 


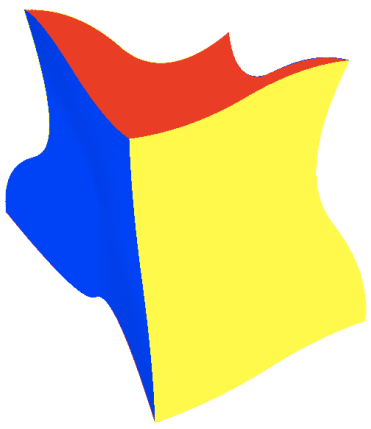

(a) boundary surfaces

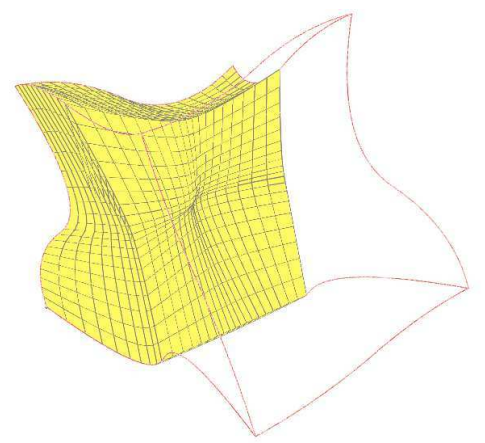

(c) Coons volume

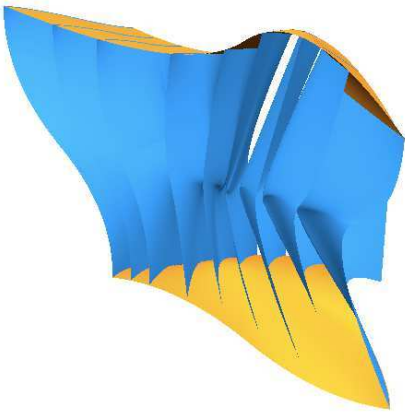

(e) initial iso-parametric surface

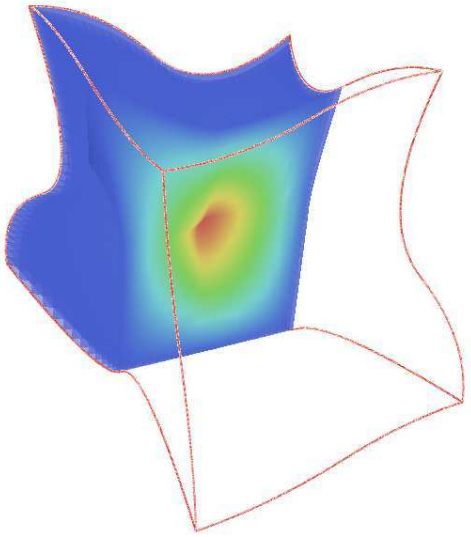

(g) solution field w.r.t Coons parameterization

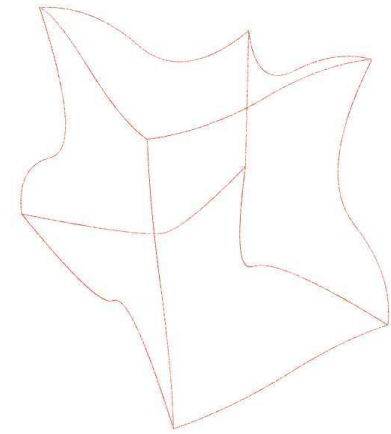

(b) boundary curves

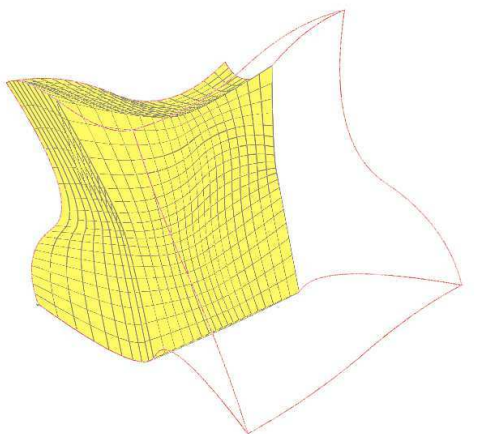

(d) optimized volume parameterization

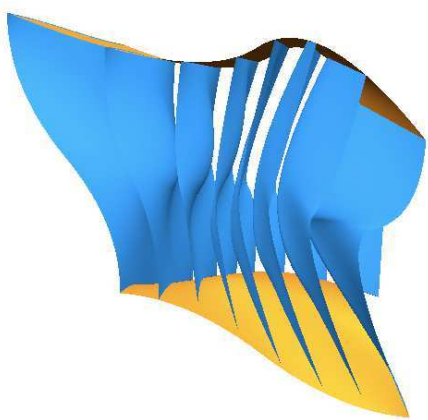

(f) optimized iso-parametric surface

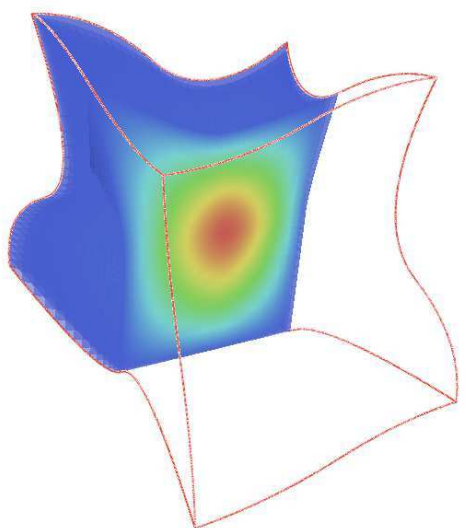

(h) solution field w.r.t optimized parameterization

Fig. 6. 3D example I. 


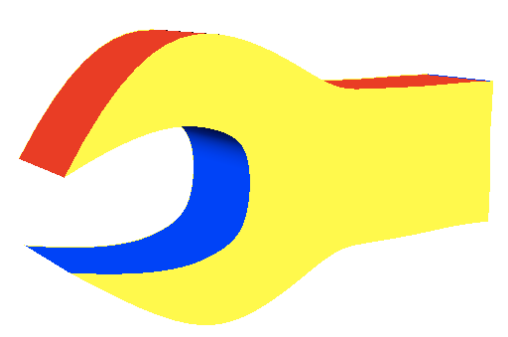

(a) boundary surfaces

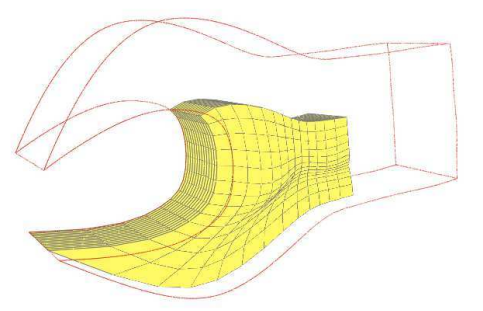

(c) Coons volume

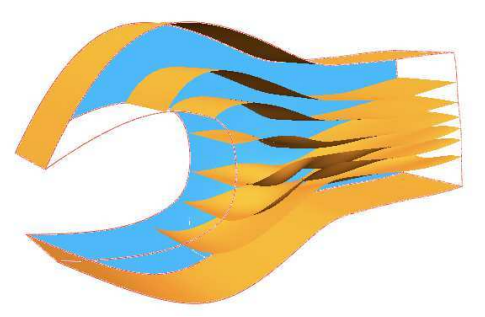

(e) initial iso-parametric surface

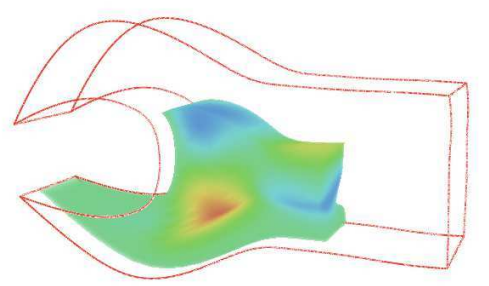

(g) solution field w.r.t Coons parameterization

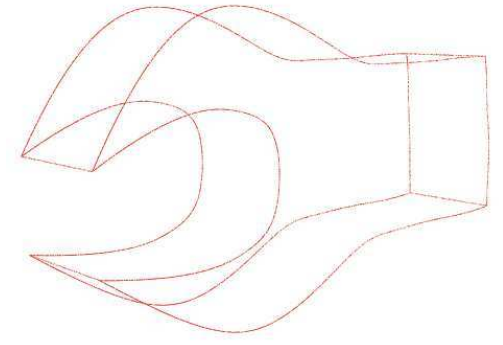

(b) boundary curves

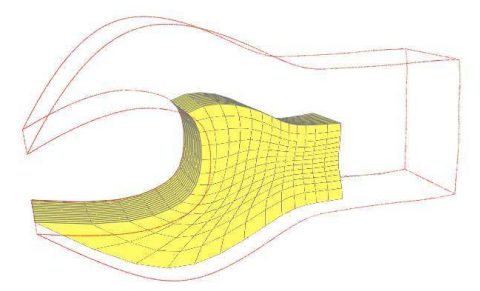

(d) optimized volume parameterization

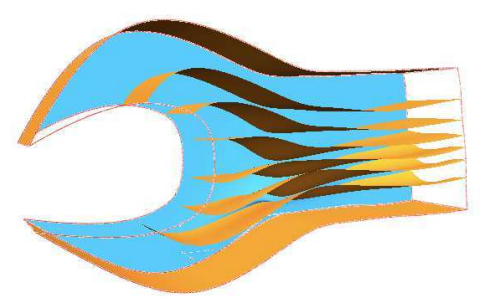

(f) optimized iso-parametric surface

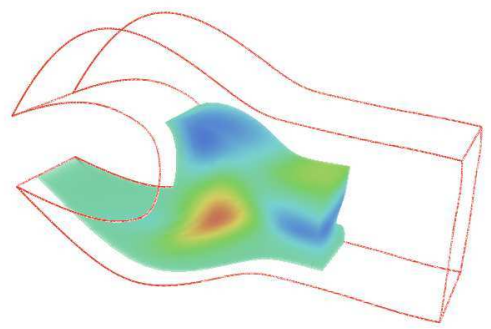

(h) solution field w.r.t optimized parameterization

Fig. 7. 3D example II. 
Table 1: Quantitative data in Fig. 3, Fig.5, Fig. 6 and Fig. 7. \# deg.: degree of B-spline parameterization; \# con.: number of control points; \# iter.: number of optimization iterations.

\begin{tabular}{cccc}
\hline Example & \# Deg. & \# Con. & \#Iter. \\
\hline Fig.3 & 3 & 36 & 4 \\
Fig.5 & 4 & 64 & 7 \\
Fig.6 & 3 & 125 & 7 \\
Fig.7 & 4 & 216 & 11 \\
\hline
\end{tabular}

\section{Conclusion}

Parameterization of computational domain is a key point in isogeometric analysis. In this paper, we propose a variational harmonic method to parameterize the $2 \mathrm{D}$ and $3 \mathrm{D}$ computational domain from the theory of harmonic mapping. The resulting parameterization is injective and has high quality near convex(concave) parts of the boundary. Examples and comparison are presented to show that the proposed methods can produce analysis-suitable parameterization of computational domain for isogeometric analysis.

As part of the future work, we will generalize the proposed method to the computational domain with complex shape, which are more important in practice.

\section{Acknowledgment}

The authors wish to thank all anonymous referees for their valuable comments and suggestions. The authors are supported by the 7th Framework Program of the European Union, project SCP8-218536 "EXCITING". The first author is partially supported by the National Nature Science Foundation of China (Nos. 61004117, 61272390, 61211130103, 61003193), the Defense Industrial Technology Development Program (A3920110002), the Scientific Research Foundation for the Returned Overseas Chinese Scholars from State Education Ministry, and the Scientific Research Starting Foundation of Hangzhou Dianzi University (No. KYS055611029).

\section{References}

[1] M. Aigner, C. Heinrich, B. Jüttler, E. Pilgerstorfer, B. Simeon and A.-V. Vuong. Swept volume parametrization for isogeometric analysis. In E. Hancock and R. Martin (eds.), The Mathematics of Surfaces (MoS XIII 2009), LNCS vol. 5654(2009), Springer, 19-44. 
[2] I. Akkermana, Y. Bazilevsa, C.E. Keesb, M.W. Farthing. Isogeometric analysis of free-surface flow. Journal of Computational Physics, 230(2011) 4137-4152.

[3] F. Auricchio, L.B. da Veiga, A. Buffa, C. Lovadina, A. Reali, and G. Sangalli. A fully locking-free isogeometric approach for plane linear elasticity problems: A stream function formulation. Computer Methods in Applied Mechanics and Engineering, 197(2007) 160-172.

[4] Y. Bazilevs, L. Beirao de Veiga, J.A. Cottrell, T.J.R. Hughes, and G. Sangalli. Isogeometric analysis: approximation, stability and error estimates for refined meshes. Mathematical Models and Methods in Applied Sciences, 6(2006) 1031-1090.

[5] Y. Bazilevs, V.M. Calo, T.J.R. Hughes, and Y. Zhang. Isogeometric fluid structure interaction: Theory, algorithms, and computations. Computational Mechanics, 43(2008) 3-37.

[6] Y. Bazilevs, V.M. Calo, J.A. Cottrell, J. Evans, T.J.R. Hughes, S. Lipton, M.A. Scott, and T.W. Sederberg. Isogeometric analysis using T-Splines. Computer Methods in Applied Mechanics and Engineering, 199(2010) 229-263.

[7] D. Burkhart, B. Hamann and G. Umlauf. Iso-geometric analysis based on Catmull-Clark subdivision solids. Computer Graphics Forum, 29(2010) 1575-1584.

[8] E. Cohen, T. Martin, R.M. Kirby, T. Lyche and R.F. Riesenfeld, Analysis-aware Modeling: Understanding Quality Considerations in Modeling for Isogeometric Analysis. Computer Methods in Applied Mechanics and Engineering, 199(2010) 334-356.

[9] J.A. Cottrell, T.J.R. Hughes, Y. Bazilevs. Isogeometric Analysis: Toward Integration of CAD and FEA. Wiley, 2009

[10] J.A. Cottrell, T.J.R. Hughes, and A. Reali. Studies of refinement and continuity in isogeometric analysis. Computer Methods in Applied Mechanics and Engineering, 196(2007) 4160-4183.

[11] J.A. Cottrell, A. Reali, Y. Bazilevs, and T.J.R. Hughes. Isogeometric analysis of structural vibrations. Computer Methods in Applied Mechanics and Engineering, 195(2006) 5257-5296.

[12] N. Crouseilles, A. Ratnani, E. Sonnendrcker. An isogeometric analysis approach for the study of the gyrokinetic quasi-neutrality equation. Journal of Computational Physics, 231(2012) 373-393.

[13] M. Dörfel, B. Jüttler, and B. Simeon. Adaptive isogeometric analysis by local h-refinement with T-splines. Computer Methods in Applied Mechanics and Engineering, 199(2010) 264-275.

[14] R. Duvigneau. An introduction to isogeometric analysis with application to thermal conduction. INRIA Research Report RR-6957, June 2009

[15] J.M. Escobara, J.M. Cascónb, E. Rodrígueza, R. Montenegro. A new approach to solid modeling with trivariate T-spline based on mesh optimization. Computer Methods in Applied Mechanics and Engineering, 200(2011) 3210-3222.

[16] G. Farin and D. Hansford. Discrete coons patches. Computer Aided Geometric Design, 16(1999) 691-700.

[17] P. Gill, W. Murray, M Wright. Practical Optimization. Springer, 1981.

[18] H. Gomez, V.M. Calo, Y. Bazilevs, and T.J.R. Hughes. Isogeometric analysis of the Cahn-Hilliard phase-field model. Computer Methods in Applied Mechanics and Engineering, 197(2008) 4333-4352.

[19] T.J.R. Hughes, J.A. Cottrell, Y. Bazilevs. Isogeometric analysis: CAD, finite elements, NURBS, exact geometry, 
and mesh refinement. Computer Methods in Applied Mechanics and Engineering, 194(2005) 4135-4195.

[20] X. Li, X. Guo, H. Wang, Y. He, X. Gu, H. Qin. Harmonic Volumetric Map- ping for Solid Modeling Applications. Proc. of ACM Solid and Physical Modeling Symposium 2007, 109-120.

[21] T. Martin, E. Cohen, and R.M. Kirby. Volumetric parameterization and trivariate B-spline fitting using harmonic functions. Computer Aided Geometric Design, 26(2009):648-664.

[22] T. Nguyen, B. Jüttler. Using approximate implicitization for domain parameterization in isogeometric analysis. International Conference on Curves and Surfaces, Avignon, France, 2010.

[23] N. Nguyen-Thanh, H. Nguyen-Xuan, S.P.A. Bordasd and T. Rabczuk. Isogeometric analysis using polynomial splines over hierarchical T-meshes for two-dimensional elastic solids. Computer Methods in Applied Mechanics and Engineering, 200(2011) 1892-1908.

[24] N. Nguyen-Thanh, J. Kiendl, H. Nguyen-Xuan, R. Wchner, K.U. Bletzinger, Y. Bazilevs, T. Rabczuk. Rotation free isogeometric thin shell analysis using PHT-splines. Computer Methods in Applied Mechanics and Engineering, 200(2011) 3410-3424.

[25] K.F. Pettersen, V. Skytt. Spline volume fairing. 7th International Conference on Curves and Surfaces. Avignon, France, June 24-30, 2010, Lecture Notes in Computer Science, 6920(2012) 553-561.

[26] A. Ratnani, E. Sonnendrcker. An arbitrary high-order spline finite element solver for the time domain maxwell equations. Journal of Scientific Computing, 51(2012) 87-106.

[27] S.P. Spekreijse. Elliptic grid generation based on Laplace equations and algebraic transformations. Journal of Computational Physics, 118(1995) 38-61.

[28] C.L. Wang, K. Tang. Non-self-overlapping Hermite interpolation mapping: a practical solution for structured quadrilateral meshing. Computer-Aided Design, 37(2005) 271-283.

[29] C.L. Wang, K. Tang. Non-self-overlapping structured grid generation on an n-sided surface. International Journal for Numerical Methods in Fluids, 46(2004) 961-982.

[30] C.L. Wang, K. Tang. Algebraic grid generation on trimmed surface using non-self-overlapping Coons patch mapping. International Journal for Numerical Methods in Engineering, 60(2004) 1259-1286.

[31] W. Wang, Y. Zhang, L. Liu, T. J.R. Hughes. Trivariate solid T-spline construction from boundary triangulations with arbitrary genus topology. Computer-Aided Design, 45(2013) 351-360.

[32] N.J. Wu, T.K. Tsay, T.C. Yang, H.Y. Chang. Orthogonal grid generation of an irregular region using a local polynomial collocation method. Journal of Computational Physics, 243(2013) 58-73.

[33] J. Xia, Y. He, X. Yin, S. Han, and X. Gu. Direct product volume parameterization using harmonic fields. Proceedings of IEEE International Conference on Shape Modeling and Applications 2010, 3-12.

[34] J. Xia, Y. He, S. Han, C.W. Fu, F. Luo, and X. Gu. Parameterization of star shaped volumes using Green's functions. Proceedings of Geometric Modeling and Processing 2010, 6130(2010) 219-235.

[35] G. Xu, B. Mourrain, R. Duvigneau, A. Galligo. Optimal analysis-aware parameterization of computational domain in 3D isogeometric analysis. Computer-Aided Design, 45(2013) 812-821.

[36] G. Xu, B. Mourrain, R. Duvigneau, A. Galligo. Parameterization of computational domain in isogeometric analysis: methods and comparison. Computer Methods in Applied Mechanics and Engineering, 200(2011) 20212031. 
[37] G. Xu, B. Mourrain, R. Duvigneau, A. Galligo. Analysis-suitable volume parameterization of multi-block computational domain in isogeometric applications. Computer-Aided Design, 45(2013) 395-404.

[38] Y. Zhang, W. Wang, T. J.R. Hughes. Solid T-spline construction from boundary representations for genus-zero geometry. Computer Methods in Applied Mechanics and Engineering, 201(2012) 185-197.

[39] Y. Zhang, W. Wang, T. J.R. Hughes. Conformal solid T-spline construction from boundary T-spline representations. Computational Mechanics, 51(2013) 1051-1059.

[40] Y. Zhang, Y. Jia, S.Y. Wang. An improved nearly-orthogonal structured mesh generation system with smoothness control functions. Journal of Computational Physics, 231(2012) 5289-5305.

[41] Y. Zhang, Y. Jia, S.Y. Wang. 2D nearly orthogonal mesh generation with controls on distortion function. Journal of Computational Physics, 218(2006) 549-571. 